\title{
La linguistique et la variété de ses grammaires
}

\section{Andrew Smith ${ }^{1}$}

Universidad Nacional, Costa Rica

\section{RESUMEN}

Este artículo presenta la complejidad del hecho gramatical en la enseñanza y el aprendizaje de una lengua extranjera. Temas tales como la dificultad de definir la gramática, la historia de la gramática, la abundancia de definiciones de la gramática, los tipos diferentes de la gramática y la diversidad de las situaciones de la enseñanza son discutidos.

\section{Résumé}

Cet article présente la complexité du fait grammatical dans l'enseignement et l'apprentissage d'une langue étrangère. Des thèmes comme la difficulté de définir la grammaire, l'histoire de la grammaire, l'abondance des définitions de la grammaire, les différents types de grammaire et la diversité des situations d'enseignement sont discutés.

Palabras clave: lingüística, definición de la gramática, historia de la gramática, tipos de gramática, diversidad de métodos sobre la enseñanza de la gramática. Mots-clés : linguistique, définition de la grammaire, histoire de la grammaire, types de grammaire, diversité de l'enseignement.

Correo electrónico: aldsmith@ racsa.co.cr 


\section{La difficulté à définir la grammaire}

Dans la situation de l'enseignement et l'apprentissage d'une langue étrangère, la présence de la grammaire est un fait indiscutable. Si la grammaire est étudiée ouvertement, d'une manière explicite ou si elle est présentée ou étudiée d'une manière implicite, elle est toujours présente. Une grande partie donc, du travail pour l'apprenant dans l'étude d'une langue étrangère est sans doute l'étude ou acquisition de la grammaire. Mais comment les enseignants, présentent-ils ou enseignent-ils la grammaire ? Quelle est leur perception de la grammaire ?Quelle est la perception de la grammaire chez l'étudiant ? La perception même de la grammaire peut varier avec chaque enseignant, chaque étudiant, chaque pays, chaque langue et chaque culture. Un enseignant forme sa perception de la grammaire de ses études personnelles et certainement de la perception de ses anciens enseignants. Si cette perception varie avec chaque langue ou culture, il peut y avoir des problèmes de standardisation de l'étude de la grammaire. La question peut même se poser "Qu'est-ce que la grammaire ? » La réponse à cette question n'est pas facile, comme Claude Germain et Hubert Séguin nous disent dans leur ouvrage Le point sur la grammaire: « Faire le point sur la grammaire n' est pas une tâche aisée car il n'existe pas une mais plusieurs interprétations de ce qu'est la grammaire ${ }^{2}$. Pierre Martinez nous dit presque la même chose dans La didactique des langues étrangères quand il écrit : « Le rôle joué par la grammaire dans la didactique des langues est des plus controversés. Une raison tient sans doute au flou de sa définition, qui recouvre la description des règles de fonctionnement général d'une langue, ou un ensemble de prescriptions imposéehus à ceux qui parlent, ou encore le système des règles de L2 intériorisées par l'apprenant $»^{3}$.

\footnotetext{
2 Claude Germain et Hubert Séguin, Le point sur la grammaire (Paris : CLE International, 1998) III.

${ }^{3}$ Pierre Martinez, La didactique des langues étrangères (Paris : PUF, 1996) 94.
} 
A vant de voir quelques définitions de la grammaire, il est utile de savoir d'où vient la grammaire et quelle est l'origine du mot, du concept, ou de la science de la grammaire.

\section{L'histoire de la grammaire}

Selon Cuq dans son livre Une introduction à la didactique de la grammaire en Français Langue Étrangère, la grammaire « fait partie des plus anciennes tentatives de connaissance de l'homme " ${ }^{4}$. L'origine du mot grammaire vient du grec « gramma » qui veut dire la lettre ou la figure ${ }^{5}()$, et ceci signifie que « Dès l'origine donc, en tout cas dans la tradition occidentale, c'est la partie écrite du langage qui a été ainsi mise en avant... " ${ }^{6}$. Les Grecs étaient les premiers à enseigner la grammaire dans une école en 335, qui se trouvait « dans le bois du Lukeion, du nom d'Apollon Lycien », d'ou vient le nom «lycée »". Dans Poétique Aristote a nommé des parties de grammaire comme le nom, le verbe, la conjonction, l'articulation. Donc, il a vraiment commencé l'étude de la grammaire, qui a été continuée par Aristarque et Apollonios.

Plus tard, les Romains Ciceron d'Arpinum et Quintilien de Calaguries ont écrit des ouvrages qui ne traitaient pas directement de la grammaire, mais qui ont été étudiés et cités plus tard par les grammairiens Varron de Rate, Saint Jerôme et Priscien de Césarée. C'est au Moyen Âge que la grammaire est considérée comme "l'un des trois arts du langage » et elle « est à la base de l'éducation " 8 . Pendant la Renaissance beaucoup de grammaires ont été écrites pour étudier les langues comme l'italien, le catalan, l'allemand, l'anglais, le basque, et bien sûr, le français. Après, au XVII ${ }^{\mathrm{e}}$ siècle, en 1660 , Amauld et Lancelot ont écrit Grammaire Générale et Raisonnée, et en

\footnotetext{
${ }^{4}$ Jean-Pierre Cuq, Une introduction à la didactique de la grammaire en Français langue étrangère (Paris : Les Éditions Didier, 1996) 7.

${ }^{5}$ Germain et Séguin, 4.

${ }^{6}$ Cuq. 7.

${ }^{7}$ Cuq, 8.

${ }^{8}$ Cuq, 11.
} 
1662 Arnauld a aussi écrit avec Nicole, La Logique ou l'art de Penser. En 1767 Beauzée a écrit Grammaire Générale, mais il paraît que la grammaire n'était pas utilisée dans l'étude des langues sinon, elle était étudiée seule. Finalement c'est au XVIII ${ }^{\mathrm{e}}$ et au $\mathrm{XIX}^{\mathrm{e}}$ siècle que la grammaire est étudiée pour l'enseignement des langues et des œuvres comme Éléments de la grammaire françoise (1780) de CharlesFrançois Lhomond, Grammaire françoise simplifiée (1778) de François Urbain Domergue, et La nouvelle grammaire française (1823) de Noël et Chapsal font leur apparition, et c'est pendant cette époque que la grammaire française commence à être enseignée comme elle est toujours enseignée aujourd'hui ${ }^{9}$.

\section{L'abondance des définitions de la grammaire}

Quand on commence à chercher une définition pour la grammaire, on constate tout de suite que trouver une définition unique n' est pas possible. Il y a une grande quantité de définitions pour la grammaire et cette réalité montre la complexité à définir ce terme. S'il n'est pas facile de donner simplement une définition pour cette discipline ou science, on peut comprendre pourquoi elle est si problématique à étudier et à enseigner. Après tout, il faut savoir ce qu'on enseigne ou étudie a vant de l'enseigner ou de l' apprendre. Et même si la grammaire est difficile à définir ou s'il existe une variété de définitions, ces définitions sont extrêmement intéressantes, voire quelquefois passionnantes. Il est intéressant de considérer quelques-unes de ces définitions de la grammaire pour voir si nous pouvons formuler une réponse ou même des réponses à cette question de « ce que c'est que la grammaire?»

La liste des différentes types de grammaire est assez longue : la grammaire classique morpho-syntaxique, la grammaire générativetransformationnelle, la grammaire notionnelle-fonctionelle, la grammaire énonciative et la grammaire textuelle. Cuq parle des écoles

9 Cuq. 11-13.

12 
de grammaire, et il nomme le distributionnalisme, le transformationalisme. l'école générativiste, le courant pragmatique et la grammaire de texte. Dans la grammaire d'aujourd'hui d'Arrivé, Gadet et Galmiche, les auteurs nomment « la grammaire fonctionnelle », et « la grammaire distributionnelle » ${ }^{10}$. Besse et Porquier parlent de « la grammaire intériorisée, » des « descriptions et simulations grammaticales » et des « modèles métalinguistiques ». Selon eux, la grammaire intériorisée est « un phénomène humain d'ordre biogénétique et psycho-social »"

\section{Les définitions des dictionnaires}

Dans le Petit Larousse Illustré nous trouvons la définition suivante : "Science des règles du langage parlé ou écrit. (La morphologie et la syntaxe sont les deux parties principales de la grammaire.) || Etude systématique des éléments constitutifs d'une langue : grammaire historique, diachronique » ${ }^{12}$. (Dans le PetitRobert 1 il y a une définition pareille, mais plus élaborée : « Ensemble des règles à suivre pour parler et écrire correctement une langue, ... - Ling. Ensemble des structures et des règles qui permettent de produire tous les énoncés appartenant à une langue et seulement eux ... V. Morphologie, syntaxe... Ling. Etude systématique des éléments constitutifs d'une langue. V. Phonétique, phonologie ; morphologie, syntaxe. La grammaire et la lexicologie, et la sémantique. - Spécialt. Étude des formes et des fonctions (morphologie et syntaxe). Grammaire descriptive ou synchronique. Grammaires structurales. Grammaire distributionnelle, par constituants immédiats. Grammaire transformationnelle, générative-transformationnelle, applicative. Grammaire historique ou diachronique. Grammaire normative,

\footnotetext{
${ }^{10}$ Michel Arrivé, Françoise Gadet et Michel Galmiche, La grammaire d'au jourd 'hui : Guide alphabérique de linguistique française (Paris : Fammarion, 1991) 298-302.

11 Henri Besse et Rémy Porquier, Grammaires et didactique des langues (Paris : Crédif. Hatier/Didier, 1991) 13-22.

12 Petit Larousse Illustré (Paris : Larousse, 1979) 481.
} 
dogmatique, ou « bon usage ». Grammaire générale. V. Linguistique. Grammaire et philologie. Agrégation de grammaire ${ }^{13}$.

\section{Les définitions des spécialistes}

Les deux définitions ci-dessus sont intéressantes parce qu'en réalité elles disent que la grammaire est simplement ce dont on a besoin pour parler et écrire une langue correctement. Mais les spécialistes en didactique des langues étrangères, comment définissentils la grammaire ? Selon Germain et Séguin, "La grammaire sera définie ici, à l'instar de Besse comme la connaissance intériorisée que possède l'usager d'une langue. Il s'agit d'une forme de compétence : la compétence grammaticale. Se représenter la grammaire comme une forme de connaissance intériorisée pose de prime abord toute la question du mode d'accès à ce type de connaissance. Là encore, tout comme Besse, nous croyons qu'il est primordial de faire la distinction entre la connaissance des aspects grammaticaux d'une langue et les tentatives de description ou de simulation qu'en font linguistes et didacticiens. Cela fait partie des ambiguïtés que renferme le terme de grammaire, tant en anglais qu'en français, en ce qu'il peut désigner tout autant une connaissance intériorisée (une compétence grammaticale) que la description ou la simulation de cette connaissance ${ }^{14}$.

Geneviève-Dominique de Salins dans Grammaire pour l'enseignement du FLE (Français Langue Etrangère) parle de la grammaire sans essayer de la définir, sinon en disant qu'il y a des notions préétablies dans la grammaire: "Le facteur grammaticolinguistique est plus difficile à cerner. En effet, les points de vue théoriques des différentes linguistes ne répondent pas nécessairement aux besoins d'apprenants étrangers venant des quatre coins du monde. Mais il est une théorie linguistique qui subsume les descriptions

\footnotetext{
13 Petit Robert 1 : Dictionnaire al phabétique et analogique de la langue française (Paris : Dictionnaires Le Rober, 1990) 882.

${ }^{14}$ Germain et Séguin, 31-32.
} 
grammaticales formelles pour atteindre le plus directement possible le sens véhiculé par l'ensemble des langues, que celles-ci soient à tons, agglutinantes ou analytiques. Cette linguistique nous importe par son approche sémantique de la grammaire. Elle recherche les notions quasi universelles qui permettent de regrouper en catégories de sens les diverses formes que la grammaire traditionnelle a pour principe de séparer. Les opérations conceptuelles qu'elle propose sont suffisamment larges pour faciliter, chez tout apprenant étranger, la saisie d'un sens global qui s'exprimera par différentes formes ${ }^{15}$.

Patrick Charaudeau dans Grammaire du sens et de l'expression ne veut pas, non plus, parler d'une définition de la grammaire mais il dit : « On le sait maintenant il n' existe pas une grammaire, ni a fortiori la grammaire d' une langue, mais autant de grammaires que de théories sur la langue. Il ne faut pas oublier que c'est le point de vue théorique qui détermine la description d'un objet, et non l'inverse. Vérité difficile à accepter, parce que la langue est le seul moyen dont nous disposons pour nous reconnaître (et être reconnu) comme appartenant à un groupe social. La langue est le garant de notre identité collective. Dès lors, comment en accepter une représentation qui ne soit pas homogène et unique ? Que cette représentation éclate en autant de descriptions que de théories et voilà notre être collectif qui se fragmente et ne se retrouve plus ${ }^{16}$.

Dominique Maingueneau choisit, dans Les Fondamentaux Syntaxe du Français, au lieu de définir la grammaire, de se concentrer sur ce qui est important, grammaticalement, pour la communication : «Décrire les catégories et les modes d'organisation essentiels, en n'hésitant pas à lier des phẹ́nomènes que la grammaire scolaire range dans des rubriques disjointes. Au lieu de s'en tenir à la classification des formes, il convient de privilégier ce qui facilite l'intelligence des fonctionnements syntaxiques majeurs... Nous avons

\footnotetext{
15 Geneviève-Dominique de Salins, Grammaire pour l'Enseignement/Apprentissage dı FLE (Paris : Didier-Hatier, 1996) 7-8.

${ }^{16}$ Patrick Charadeau, Grammaire du sens et de l'expression (Paris : Hachette, 1992) 3.
} 
donc dû nous résigner aux simplifications et aux raccourcis qui nous ont semblé les moins dommageables pour la formation grammaticale ${ }^{17}$.

Besse et Porquier parlent des descriptions et simulations grammaticales qui sont « la connaissance méthodique et explicite que les grammairiens et linguistes élaborent à partir de l'observation des manifestations d'une grammaire intériorisée particulière ${ }^{18}$, et ils continuent avec les modèles métalinguistiques qui sont « l'ensemble des concepts et des raisonnements à partir desquels le linguiste ou le grammairien cherche à décrire ou à simuler la grammaire intériorisée commune d'un sous-ensemble des sujets parlant une langue ${ }^{19}$.

En somme, ces spécialistes ne veulent pas définir la grammaire et s'ils le font, ils le font d'une manière diverse et ils parlent des règles, quelque fois des connaissances intériorisées, d'une compétence et des théories. La grammaire n'est pas facilement définie parce qu'il y a différentes perceptions sur le thème. On peut, par contre, voir la différence des points de vue sur la grammaire qui peuvent expliquer pourquoi il n'y a pas une seule mais plusieurs définitions pour la grammaire.

En réalité toutes ces définitions et catégories compliquées ne sont pas nécessaires pour cette étude. Ce qui est important ici est ce que Germain et Séguin appellent « la grammaire pédagogique » et qui peut être appelée tout simplement la grammaire d'une langue étrangère ${ }^{20}$. C'est la grammaire ou les points grammaticaux nécessaires pour l'apprentissage d'une langue. En français, par exemple, il y a les articles définis, indéfinis, la conjugaison des verbes au présent, les pronoms personnels, etc. Par exemple, dans son livre Grammaire pour l'Enseignement/Apprentissage du FLE, Geneviève-Dominique de Salins présente 10 dossiers qui nous donnent une idée des catégories grammaticales nécessaires dans l'étude du FLE: La présentation, la

\footnotetext{
17 Dominique Maingueneau, Les Fondametaux - Syntaxe du Français (Paris : Hachette, 1994) 3-4.

18 Besse et Porquier, 16.

19 Besse et Porquier, 22.

20 Germain et Séguin, 85.
} 
personne, l'actualisation du nom commun, l'affirmation et la négation, l'interrogation et la forme interrogative, la qualification, la situation dans l'espace, la situation dans le temps et la version du procès, l'argumentation et opérations logiques, l'actance et les rôles actanciels ${ }^{21}$. Unautre exemple d'un texte de grammaire La Grammaire à l'auvre, de John Barson a 14 chapitres qui nous donnent une autre idée des catégories grammaticales qui existent dans l'étude de la langue française : «Le présent et l'impératif », " Les déterminants », "Le passé », "L'interrogation", "Le futur et le conditionnel », "Les pronoms », "Les verbes pronominaux », « La négation », « Le genre et le nombre », "Le subjonctif », "La comparaison », «Les propositions relatives", "Le Discours indirect» et "Les temps littéraires ${ }^{22}$. Ces deux œuvres sont représentatives de la plupart des manuels de grammaire, c'est-à-dire, que la manière de nommer les points grammaticaux peut changer ou la manière de les classer peut changer, mais les points grammaticaux qu'il faut étudier et comprendre pour parler une langue, ne changent pas. La définition donnée par Germain et Séguin présente la grammaire comme une simulation d'une compétence humaine, une " connaissance intériorisée " que chacun possède. La définition de Salins présente la grammaire comme un métalangage catégoriel, des groupements de catégories pour séparer les formes. De plus, Charaudeau présente « les » grammaires comme descriptions de systèmes de langues différentes et comme des prescriptions d'usages sociaux propres à chaque langue. Il y a même le point de vue où l'on considère les grammaires comme le produit commercial qui est en vente concurrentielle sur le marché des langues. Arrivé, Gadet et Galmiche parlent des « manuels, recueils et essais » de grammaire, encore une autre façon de définir la grammaire ${ }^{23}$.

\footnotetext{
21 de Salins, 3-4.

22 John Barson, La Grammaire à l'acuvre, 4e éd. (New York : CBS College Publishing, 1986) v-xi.

${ }^{23}$ Michel Arrivé, Françoise Gadet et Michel Galmiche, La grammaire d'aujourd' hui : Guide al phabétique de linguistique française (Paris : Fammarion, 1991).
} 


\section{Les différents types de grammaire}

Dans la liste dejà citée de différents types de grammaire dans le Petit Robert 1 on voit bien la quantité de types de grammaires reconnues dans la didactique. De même, dans Une introduction à la didactique de la grammaire en Français langue étrangère, JeanPierre Cuq mentionne la grammaire comparée, la grammaire historique, le distributionnalisme, le transformationalisme, la grammaire générative et la grammaire de texte. Dans la grammaire d'aujourd'hui on trouve la grammaire dite "traditionnelle » et les grammaires d'inspiration linguistique ainsi que la grammaire générative ${ }^{24}$.

\section{La classification de Dirven}

Pour comprendre comment essayer de décrire la connaissance intériorisée d'une langue aux apprenants, il faut d'abord comprendre les types de grammaire dont on parle quand on étudie une langue étrangère. La classification de Dirven sépare la grammaire pédagogique de la grammaire descriptive avec des sous-classifications qui souvent coïncident. Pour cet auteur, la grammaire est définie tout simplement comme «un ensemble de règles ${ }^{25}$. Pour l'enseignement / apprentissage des langues, on peut tirer quelques catégories de cette classification étant les plus importantes : la grammaire de l'usager, la grammaire de l'enseignant, et la grammaire de l'apprenant ${ }^{26}$.

La grammaire de l'usager, ou la connaissance intériorisée de sa langue maternelle que chaque personne possède, est importante parce que c'est la grammaire que l'apprenant va utiliser pour apprendre ou assimiler la grammaire de la langue-cible.

Le deuxième type, la grammaire de l'enseignant, est la connaissance que l'enseignant possède de la langue enseignée. Cet

\footnotetext{
24 Arrivé, Gadet et Galmichet.

${ }^{25}$ Germain et Séguin, 48.

${ }^{26}$ Germain et Séguin, 49.
}

18 
enseignant peut être un natif, qui enseigne sa langue maternelle, ou un étranger qui enseigne sa deuxième langue.

Finalement, la grammaire de l'apprenant est l'interlangue grammaticale de l'apprenant qui est en développement constant ${ }^{27}$.

La représentation que chaque apprenant a de la grammaire va directement liée avec les classifications de la grammaire. D'autre part, la perception que l'enseignant possède de la grammaire est ce qu'il/ elle va essayer de présenter aux apprenants. Cette idée est complexe, parce qu'on ne sait jamais si l'enseignant va pouvoir enseigner sa représentation personnelle de la grammaire, et si sa conception de la grammaire va influer sa manière d'enseigner la grammaire ${ }^{28}$. L'apprenant normalement, lui aussi, possède une représentation de la grammaire, et l'enseignant a le devoir de, non seulement présenter sa représentation de la grammaire, mais aussi le faire de façon que l'apprenant lui même puisse la rapprocher de sa représentation personnelle de la grammaire.

La classification grammaticale de Dirven a été adaptée pour mieux représenter la grammaire nécessaire dans la didactique des langues. Dans cette classification adaptée il y a une grammaire d'enseignement, une grammaire d'apprentissage, une grammaire de référence, une grammaire linguistique, et une grammaire pédagogique. Chacune d'elles est censée de présenter un aspect de la grammaire ${ }^{29}$.

La grammaire d'enseignement, comme le nom l'indique, est la grammaire qui est choisie pour être utilisée dans l'enseignement d'une langue étrangère et elle est normalement présentée sous la forme d'un guide pour l'enseignant (livre du professeur) ou par un programme préparé par l'institution où elle est enseignée. La grammaire d'apprentissage est présentée comme un livre ou cahier que l'apprenant utilise pour apprendre la grammaire. La grammaire de référence, un mélange de la grammaire pédagogique et la grammaire linguistique,

\footnotetext{
27 Germain et Séguin, 49.

28 Germain et Séguin, 49.

29 Germain et Séguin, 53-54.
} 
est un livre de référence pour présenter et expliquer la grammaire. La grammaire linguistique, pareille à la grammaire pédagogique, est une description plus « scientifique » de la grammaire, une explication plus profonde, qui n'est pas nécessairement ce dont on a besoin dans l'enseignement didactique d'une langue, si le but est de parler et d'écrire la langue. Finalement, la grammaire pédagogique, est la grammaire nécessaire pour parler et écrire la langue, la grammaire pour l'enseignant et l'apprenant. Elle est présentée comme des «directives pédagogiques ${ }^{30}$.

On constate immédiatement que ces définitions de la grammaire se ressemblent souvent et que souvent une définition de la grammaire comprend une partie d'une autre définition. Donc, une grammaire d'enseignement vue dans un livre du professeur, par exemple, est en réalité une grammaire pédagogique. Ceci est normal, parce qu'en réalité chaque définition ou perception de la grammaire est un essai de définition de la grammaire et « Il y a autant de grammaires qu'il y a de points de vue sur l'objet ; tout comme il y a autant de descriptions ou simulations linguistiques, qu'il y a d'écoles de pensée linguistique » 31 . Le lien entre « ces grammaires » ou « la grammaire » et les différentes méthodologies, est un lien fort et intéressant. «La grammaire » a existé avant « les méthodologies », mais les méthodologies essaient d'utiliser d'une manière ou d'une autre, la grammaire pour enseigner la langue. Est-ce que les méthodologies d'enseignement définissent la grammaire, ou est-ce que la grammaire définit les méthodologies ? La méthode traditionnelle utilise, en théorie, la grammaire pour enseigner la langue donc cette grammaire serait une grammaire pédagogique. Mais une méthode comme la méthode naturelle qui maintient que « la grammaire sera acquise (au lieu d'être apprise) essentiellement par l'intermédiaire des inputs de compréhension » chercherait plutôt à développer la grammaire intériorisée de l'apprenant ${ }^{32}$.

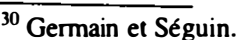

${ }^{31}$ Germain et Séguin, 59.

${ }^{32}$ Bernard Dufeu, Les approches non conventionnelles des langues étrangères (Paris : Hachette, 1996) 106.
} 


\section{La diversité des situations d'enseignement}

S'il n' y a pas de réponse facile pour la définition de la grammaire, il ne peut pas y avoir de réponse facile à la question de comment enseigner la grammaire. La question devient même plus difficile si nous considérons la réalité de l'enseignement des langues étrangères et secondes dans le monde. Il y a des professeurs natifs et non-natifs enseignant des langues avec des formations, perceptions et manières d'enseigner variées. Il est difficile de savoir ce que l'enseignant fait dans son cours, parce que normalement personne n'a accès au cours d'un professeur. Alors, même si le professeur a un bon programme on ne sait pas s'il le suit en pratique, ou comment il le suit. Et il existe toujours la question de que c'est qu' un bon programme de grammaire.

On doit aussi prendre en compte la réalité des différents types d'établissements où l'on enseigne les langues. Il y a des universités publiques et privées, des lycées, l'Alliance Française, des instituts de langues privées, chacun avec ses différents cours, ses politiques et ses restrictions. Alors, le problème de comment enseigner la grammaire et les langues en général est compliqué simplement parce que beaucoup de gens le font dans beaucoup de situations ou réalités différentes, et il est difficile de vraiment dire quelle est la meilleure méthodologie ou système d'enseigner ou de présenter la grammaire.

La difficulté de définir la grammaire, l'abondance de ses définitions avec les différents types de grammaire et la diversité des situations de son enseignement ne diminue en rien la beauté de son histoire et du fait que la grammaire aura toujours un rôle important dans l'enseignement et l'apprentissage d'une langue étrangère. 